\title{
Poliposis linfangiomatosa de amígdalas palatinas y adenoides: Reporte de un caso
}

\author{
Lymphangiomatous polyps of adenoids and palatine tonsils: One case report
}

\author{
Simona Minzer1, Lara Fernández², Gustavo Bravo², Iván Gallegos³.
}

\begin{abstract}
RESUMEN
Los pólipos linfangiomatosos son malformaciones congénitas de tipo hamartomatosas caracterizados histológicamente por una proliferación linfática vascular con distintos grados de componente fibroso, adiposo y linfático, cubiertos por un epitelio escamoso. Dado a que se conocen por distintos nombres en la literatura, sólo se han descrito alrededor de 30 casos de pólipos linfagiomatosos como tal. De etiopatogenia desconocida, se presentan como una masa polipoidea o papilomatosa en las amígdalas palatinas, con sintomatología variable. Su diagnóstico definitivo es histológico tras una resección completa. No se han reportado casos de malignización ni recurrencia. En el presente trabajo se reporta el caso de un paciente de 5 años con historia de crecimiento amigdalino bilateral de dos años de evolución. La biopsia definitiva demuestra una poliposis linfangiomatosa de amígdalas palatinas y adenoides.
\end{abstract}

Palabras clave: Pólipos, hamartoma, amígdala palatina, adenoides

\begin{abstract}
Lymphangiomatous polyps are hamartomatous congenital malformations. They are histologically characterized by a vascular lymphatic proliferation associated with fibrous, adipose and lymphatic components covered by squamous epithelium. There are only 30 cases described in the literature by the name of lymphangiomatous polyp, since it has multiple denominations. Even though their etiopathogenesis is unknown, their clinical presentation is described as a polypoid mass in the palatine tonsils, which may have multiple manifestations. The diagnosis is made histologically after complete resection. There have not been reports of malignant transformation nor recurrence. We present a case of a five year old patient with history of bilateral palatine tonsil growth. Final biopsy described lymphangiomatous polyps of adenoids and palatine tonsils.
\end{abstract}

Key words: Polyps, hamartoma, palatine tonsils, adenoids.

\footnotetext{
Interna Universidad de los Andes.

2 Médicos Servicio ORL, Hospital Clínico Universidad de Chile.

Médico Anátomo Patólogo, Hospital Clínico Universidad de Chile.
} 


\section{INTRODUCCIÓN}

El pólipo linfangiomatoso se clasifica como una malformación congénita de tipo hamartomatosa ${ }^{1}$. Los hamartomas son crecimientos simples y espontáneos derivados del tejido local, presentando un crecimiento autolimitado². Son raros en la región de cabeza y cuello ${ }^{3}$, sin embargo, éste es el sitio más frecuente de presentación de los pólipos linfangiomatosos, principalmente en piel y tejidos subcutáneos. Se encuentran bajo distintos nombres en la literatura, por lo que solo se han reportado alrededor de 30 casos de pólipos linfangiomatosos como tal, con una descripción histológica específica ${ }^{4}$. A continuación se describe un caso que se presentó en el Hospital Clínico Universitario de la Universidad de Chile.

\section{CASO CLÍNICO}

Paciente de 5 años, sin antecedents mórbidos ni quirúrgicos, consulta por roncopatía asociada a pausas respiratorias objetivadas por su madre. Refiere además un crecimiento amigdalino bilateral de dos años de evolución. Al examen físico se aprecia una hiperplasia amigdalina grado III bilateral simétrica, ambas amígdalas con superficie de aspecto papilomatoso. No se aprecian otras lesiones de este tipo al examen físico. Se realiza nasofibroscopía que objetiva una hiperplasia adenoidea grado III según la escala de Parikh, de aspecto normal. No se aprecian otras lesiones en vía aérea. Se decide realizar adenoamigdalectomía electiva.

En el intraoperatorio se procede a realizar adenoidectomía por legrado con adenótomo, donde se aprecia adenoides muy adherido a pared faríngea posterior que sangra algo más de lo habitual. Hemostasia con tórula embebida en subgalato de bismuto. Posteriormente se realiza amigdalectomía con asa fría previa disección del lecho con tijera. Hemostasia con subgalato de bismuto y bipolar. Revisión de lechos con hemostasia adecuada. Se envían muestras a biopsia diferida.

Se procesan tres muestras en el Servicio de Anatomía Patológica. Las dos amígdalas palatinas se describen como masas ovoides de superficie rosada, lobulada e irregular, con abundantes proyecciones digitiformes. La amígdala derecha mide $3 \times 2,4 \times 2 \mathrm{~cm}$ y la amígdala izquierda 2,5 × 2,2 × 1,4 cm (Figura 1). Al examen histológico se observan ambas amígdalas palatinas con superficie verruciforme y prolongaciones polipoides papilomatosas compuestas por epitelio de revestimiento escamoso maduro, no queratinizado, con moderada linfocitosis, similar al epitelio normal. El estroma es de predominio linfoide, con folículos hiperplásicos que presentan polarización, macrófagos con cuerpos tingibles y mantos conservados. Entre ellos se disponen haces irregulares de tejido conectivo denso, con vasos sanguíneos algo irregulares, dilatados, la mayoría de aspecto linfático, con ausencia de contenido hemático (Figura 2).

La tercera muestra corresponde al tejido adenoideo en varios fragmentos irregulares, rojizos, que en conjunto miden $3 \times 2,2 \times 0,7 \mathrm{~cm}$. Microscópicamente se observan fragmentos de mucosa rinosinusal con revestimiento en partes respiratorio y en partes escamoso, no queratinizado. No se observan atipias. El estromal es linfoide con prominente presencia de folículos similares a las

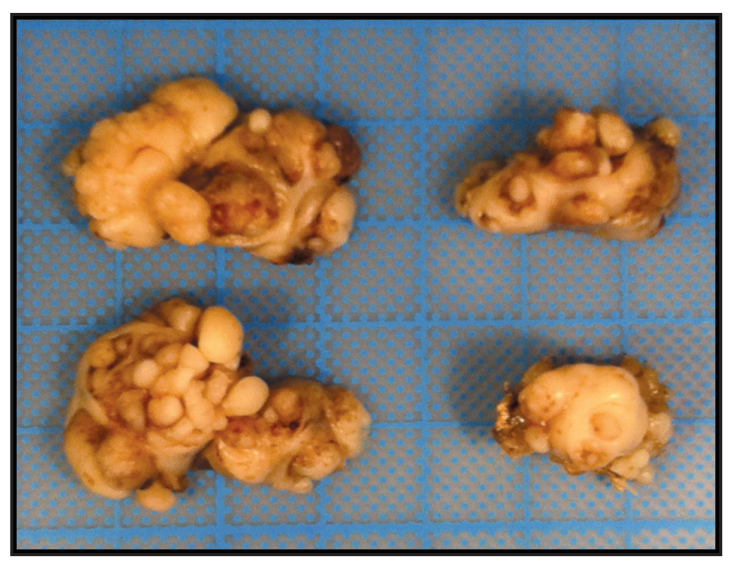

Figura 1. Muestra macroscópica: amígdalas palatinas. 


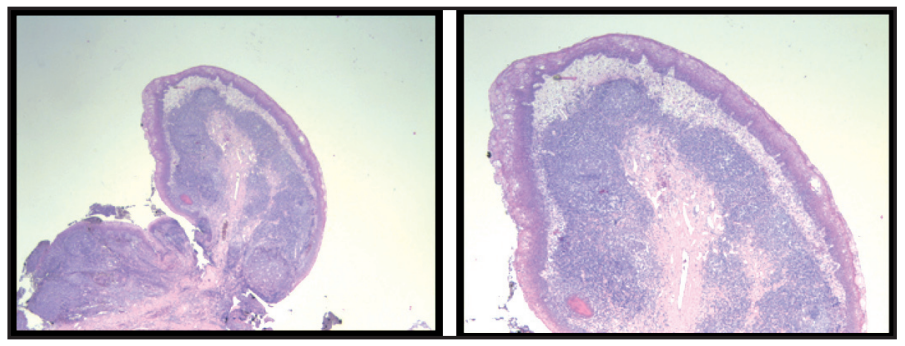

Figura 2. Corte histológico de proyección "papilomatosa" de amígdala palatina. Superficie con epitelio escamoso maduro, no queratinizante. Estroma subyacente de tipo linfoide con folículos y centros germinales. En su espesor, vasos linfáticos dilatados (Hematoxilina-Eosina, x40 y $\mathrm{x} 100)$.

amígdalas palatinas. Se observan folículos heterogéneos de tejido fibroconectivo vascularizado con vasos sanguíneos dilatados, de aspecto linfático.

El diagnóstico final histológico corresponde a una poliposis linfangiomatosa de amígdalas palatinas y adenoides.

\section{DISCUSIÓN}

Al enfrentarse a una lesión papilomatosa de las amígdalas palatinas es importante tener en cuenta que las lesiones malignas son más frecuentes que las benignas ${ }^{4}$. El llamado pólipo linfangiomatoso es una lesión benigna categorizada como hamartoma ${ }^{5}$. Se describe como un tumor congénito inusual del sistema linfático, en el que se observa una proliferación linfática vascular con distintos grados de componente fibroso, adiposo y linfático, cubierto por un epitelio escamoso $0^{1,6}$. Se observan principalmente en niños y adultos jóvenes, con una edad promedio que oscila en distintas series entre 21,2 y 29 años ${ }^{1,4,6}$. Se ha descrito que ya estarían presentes al nacimiento pero no se hacen sintomáticas hasta años más tarde. En general se describe que no hay predilección por sexo, solo un estudio mostró mayor frecuencia en hombres en una relación de 3:11'. Se ha reportado una incidencia de 1,9\%, sin embargo se piensa que puede ser mayor debido a la gran variedad de nomenclatura utilizada para referirse a este tipo de lesión ${ }^{4,6-9}$, tales como angioma, linfangiomapolipoideo, pólipo hamartomatoso, pólipo linfoide, pólipo linfangiomatoso, pólipo fibroso linfangiectásico, linfangiomapolipoideo, papiloma escamoso pedunculado, entre otros ${ }^{4}$.

Su etiopatogenia es desconocida ${ }^{1,7}$. Se ha postulado que puede tratarse de una inflamación crónica por obstrucción del drenaje linfático, sin embargo, es poco probable ya que esta lesión no se presenta en todos los pacientes con amigdalitis crónica y no todos los pacientes con pólipos linfangiomatosos tienen antecedentes de amigdalitis crónica 0 a repetición $n^{7,9,10}$. Lo más aceptado actualmente es que se trata de una proliferación de tipo hamartomatosa.

Se presenta como una masa amigdalina generalmente polipoidea y pediculada, unilateral sin predilección por un lado en especial6,9. Puede ser asintomática durante un largo período de tiempo y los casos en la literatura demuestran ser muy variables en cuanto al tiempo de evolución de la sintomatología. Los síntomas más frecuentemente descritos en Ios casos reportados fueron: disfagia, dolor de garganta, sensación de cuerpo extraño faríngeo, disnea, amigdalitis aguda unilateral, halitosis, roncopatía y apnea del sueño, rinolalia, salivación excesiva, entre otros $^{1,4-6}$. No se observó presencia de adenopatías cervicales ${ }^{2,7,10}$. En cuanto a su tamaño, pueden permanecer estáticos 0 crecer rápidamente por fenómenos de tipo hemorrágicos 0 inflamatorios ${ }^{10}$.

El diagnóstico definitivo es histológico. El pólipo linfangiomatoso amigdalino se caracteriza por presentar una proliferación al azar de distintos componentes estromales normales de la amígdala. Se observa un epitelio escamoso estratificado con hiperqueratosis asociado a un estroma compuesto por tejido conectivo laxo, fibroso y/o adiposo, agregados de tejido linfático y canales linfáticos dilatados, de paredes epiteliales finas, estructuras valvulares y contenido luminal proteináceo y linfocítico, sin evidencias de atipia 0 malignidad $4,6,7,9,10$. Como están formados por componentes normales de la amígdala, los autores piensan que se trataría de un hamartoma en vez de un linfangioma propiamente tal ${ }^{6}$. Sus canales linfáticos no son tan prominentes como los del linfangioma típico y presentan folículos linfoides más prominentes, lo que no es característico de los pólipos papilares linfoides ${ }^{6}$.

La tinción con inmunohistoquímica demostró que 
Ios vasos linfáticos contenían una pequeña pared de músculo liso y eran reactivos al antígeno relacionado al factor VIII. En una serie se tiñó a los linfocitos presentes con tinción S-100 resultando positivo. Esto demuestra una inmuno-positividad nuclear de los linfocitos, lo cual es importante ya que los linfocitos con S-100 positivos están normalmente presentes en tejido amigdalino normal y en sangre periférica y raramente en vasos linfáticos y otros órganos ${ }^{11}$. Esto apoya la teoría de que se trata de un hamartoma.

El manejo es con excisión completa y amplia de la lesión, con tendencia variada a realizar biopsia excisional 0 amigdalectomía unilateral entre los autores ${ }^{1,3,4,9,10}$. No se han reportado casos de malignización ni de recurrencia, con tiempo variable de seguimiento ${ }^{1,4,7}$. No parecen asociarse a otras malformaciones linfáticas sincrónicas como parte de un proceso generalizado, al ser estudiadas mediante técnicas imagenológicas ${ }^{9}$. Se ha sugerido que puede existir un grado de reemplazo o metaplasia de tejido adiposo a causa de isquemia del estroma y que esto puede aumentar con el tiempo, sin ninguna significancia clínica ${ }^{11}$.

Es importante tener en cuenta la gran cantidad de diagnósticos diferenciales existentes, entre ellos los linfangiomas, papilomas, pólipo fibroepitelial, neoplasia maligna, inflamación aguda o crónica, masa parafaríngea que desplaza a la amígdala, hemangiomas, angiofibroma juvenil, otras malformaciones arteriovenosas, linfangiectasia, papiloma escamoso, entre otras ${ }^{4,9,10}$.

\section{CONCLUSIONES}

Es importante tener presente el diagnóstico de pólipo linfangiomatoso al momento de enfrentarse a una lesión de la amígdala palatina. A pesar de ser una malformación benigna sin riesgo de malignización, es importante resecar la lesión, incluso en su etapa asintomática, ya que dentro de los diagnósticos diferenciales se debe contemplar una posible neoplasia. A futuro, sería prudente unificar criterios para así poder clasificar adecuadamente este tipo de lesiones.

\section{BIBLIOGRAFÍA}

1. Balatsouras D, Fassolis A, Koukoutsis G, Ganelis $P$, Kaberos A. Primary Lymphangioma of the Tonsil: A Case Report. Case Reports in Medicine 2011; ID 183182.

2. Al-Qudehy Z, Al-Nufaily Y, Yagi H. Tonsilar lymphangiomatous polyp. A case report and literatura review. The Internet Journal of Otorhinolaryngology 2013: 15(1).

3. Abu Shara K, Al-Muhana A, Al-Shennawyt M. Hamartomatous tonsilar polyp. The Journal of Laryngology and Otology 1991; 105: 1089-90.

4. Peker B, Acar M, Giritli E. A pedunculated lymphangiomatous polyp of the palatine tonsil. A case report. Braz J Otorhinolaryngol 2013; 79(3): 402.

5. Khazaei S, Emami M, Kanani M, Najafi K. Lymphangiomatous polyp of the tonsil. Pathology 2009; 41: 62.

6. Kardon D, Wenig B, Heffner D, Thompson L. Tonsillar Lymphangiomatous Polyps: A clinicopathologic series of 26 cases. Mod Pathol 2000; 13(10): 1128-33.

7. Mane V, Joshi R, Patil P. Tonsilla Lymphangioma. Indian Medical Gazette, Octubre 2012.

8. Suk Ryu H, Jung S, Koh J, Lee S. Tonsillar Lymphangiomatous Polyp: Report of two cases. The Korean Journal of Pathology 2006; 40: 381-4.

9. Park W, Pransky S, Malicki D, Hong P. Unilateral Lymphangiomatous Polyp of the Palatine Tonsil in a Very Young Child: A Clinicopathologic Case Report. Case Reports in Pediatrics 2011.

10. Coscarón E, Martin E, Ursúa I. Linfangioma Amigdalino Polipoide. Revista de la Sociedad Otorrinolaringológica de Castilla y León, Cantabria y la Rioja 2011; 2(11).

11. Ohtsuki $Y$, Kurita N, Iguchi M, Kurabayashi $A$, Matsumoto M, Takeuchi T, Furihata M. Case Report: A pedunculated hamartomatous polyp of the palatine tonsil. Biomedical Research 2006; 17(3): 155-8.

Dirección: Gustavo Bravo C

Servicio Otorrinolaringología, Hospital Clínico Universidad de Chile

Santos Dumont 999, Santiago de Chile

E mail: guztab@gmail.com 